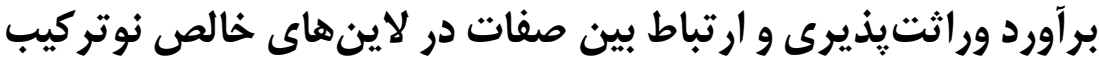

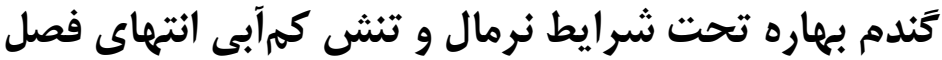

حمزه حمزه'، على اصغرى '، سيد ابولقاسم محمدى "، اميد سفاليان ع و سليمان محمدى

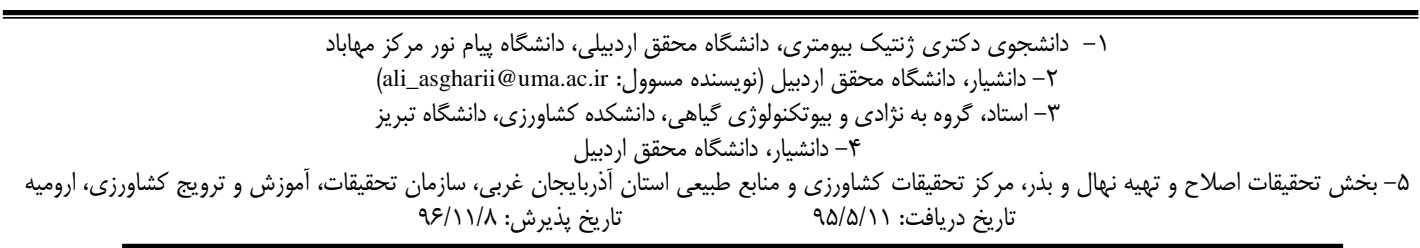

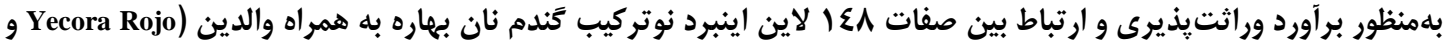

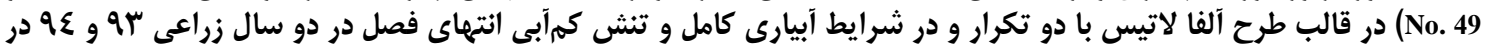

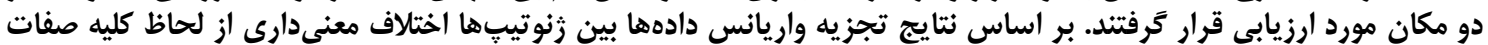

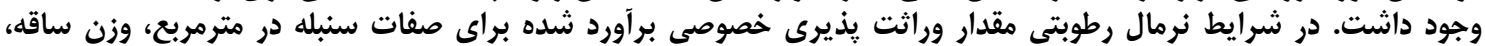

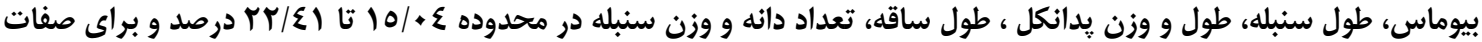

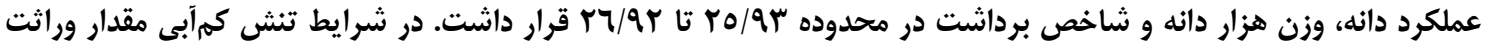

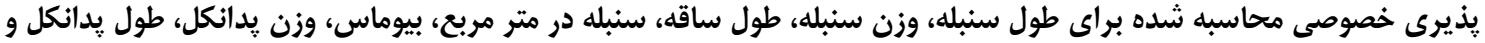

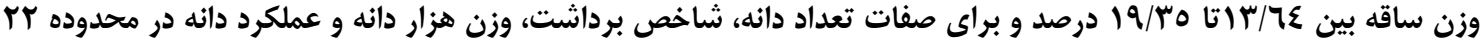

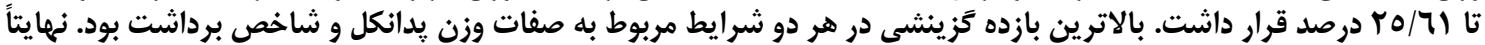

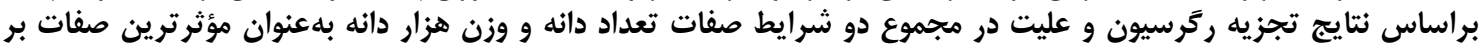

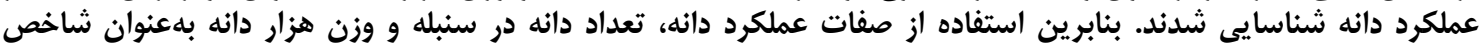

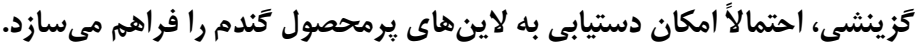

$$
\text { وازههاى كليدى: لاين اينبرد نوتركيب، وراثتيذيرى، همبستكى، كَندم نان }
$$

از رسيدن به هموزيخوتى، داراى تركيبات متفاوت از زنهاى مناى

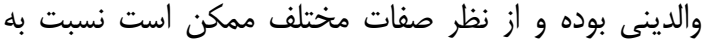

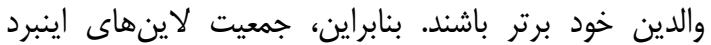

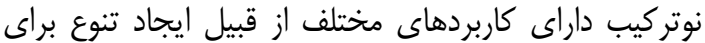

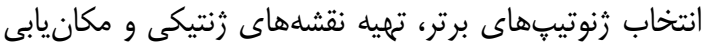

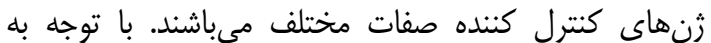

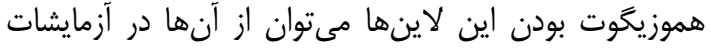

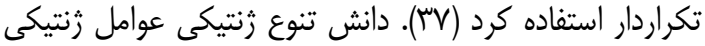

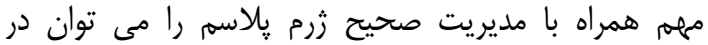

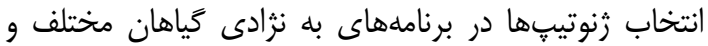

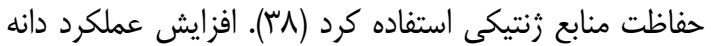

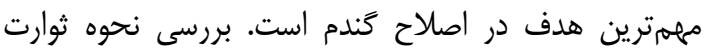

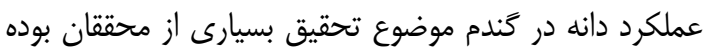

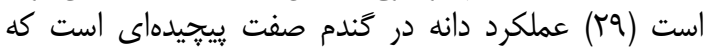

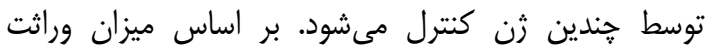

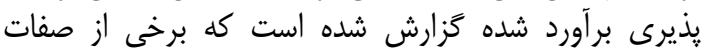

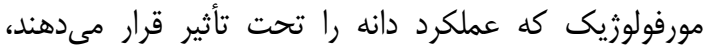

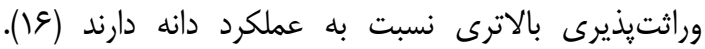

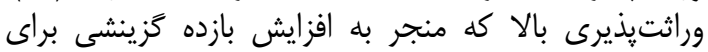

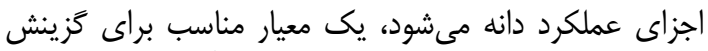

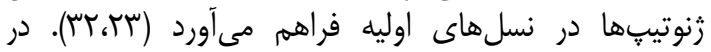

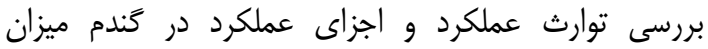

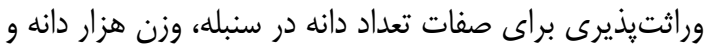

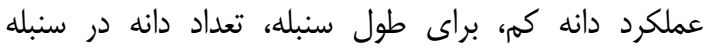
متوسط و تعداد سنبلجه در سنبله و تعداد ينجه بارور بالا دان

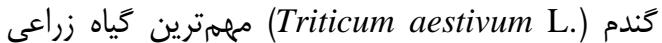

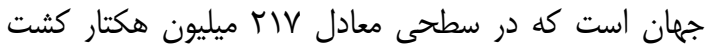

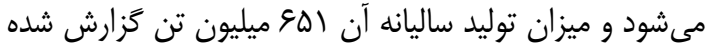

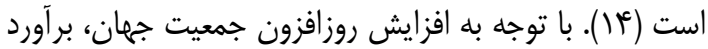

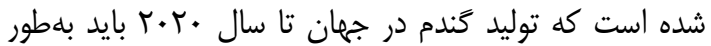

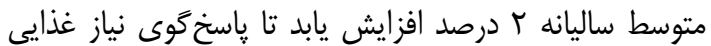

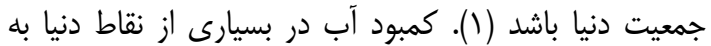

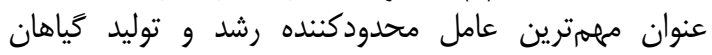

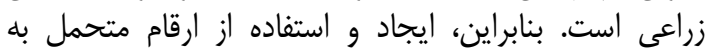

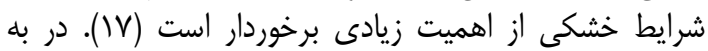

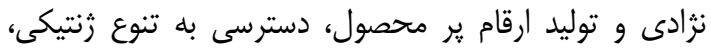

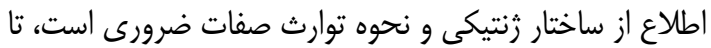

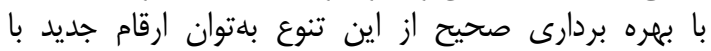

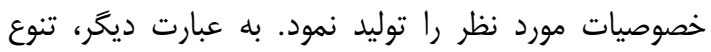

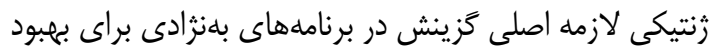

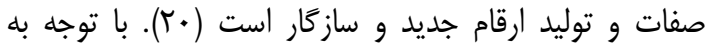

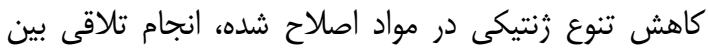

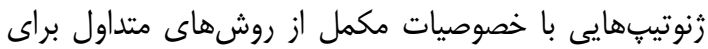

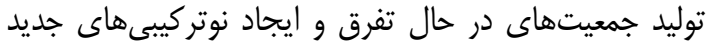

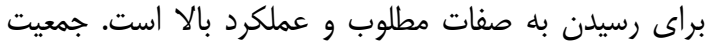

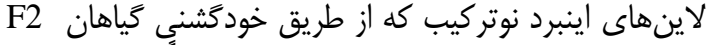

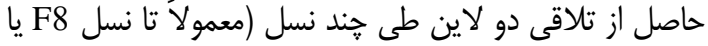

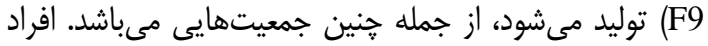

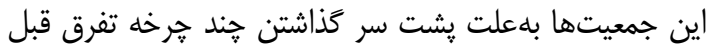


قالب طرح آلفا لاتيس با دو تكرار و در دو شرايط عادى و رون

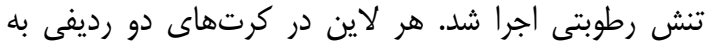

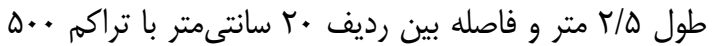

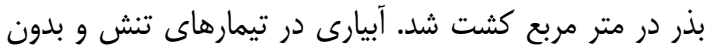

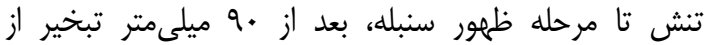

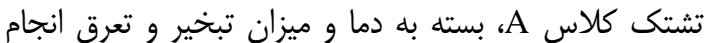

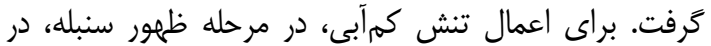

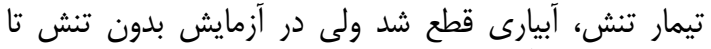

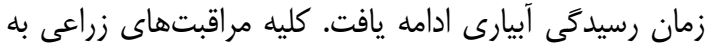

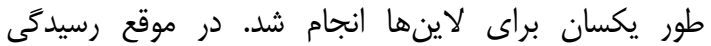

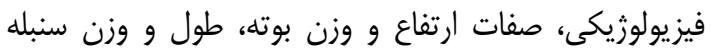

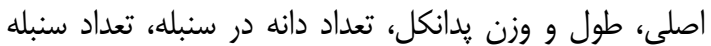

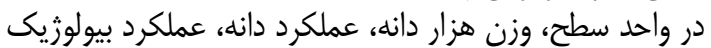

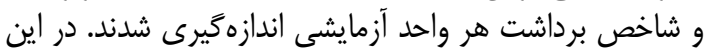

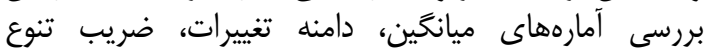

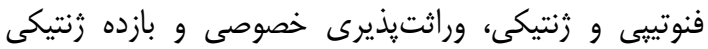

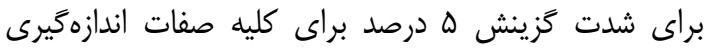

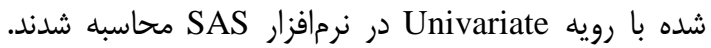

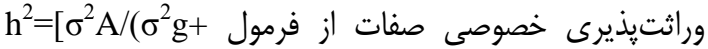

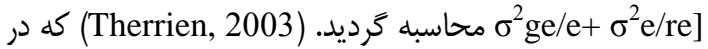
آنها

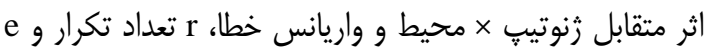

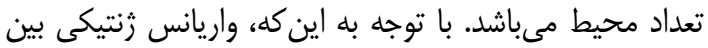

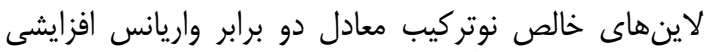

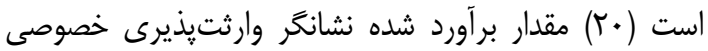

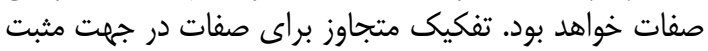

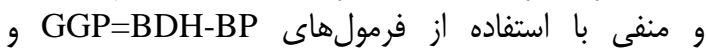
GGN GGP محاسبه كرديد كه در آن GGN=WDH-WP

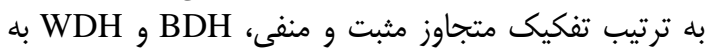

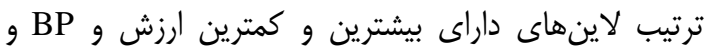

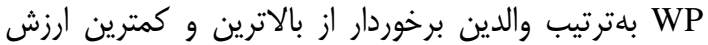

ضرايب تنوع فنوتيبى و زنوتيبى با استفاده از فرمولهاى

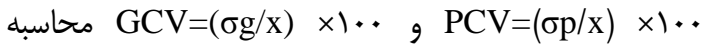

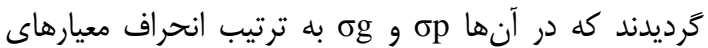

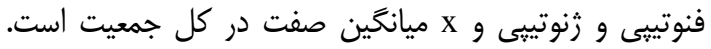

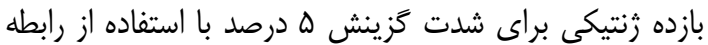

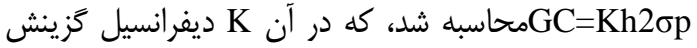

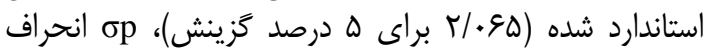

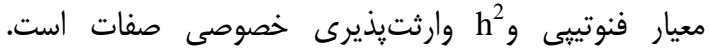

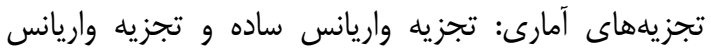

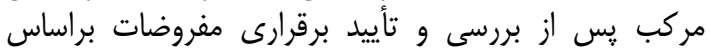

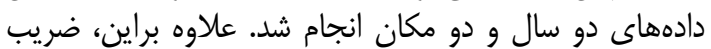

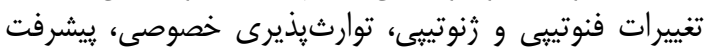

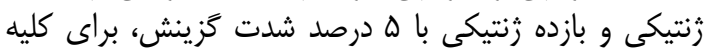

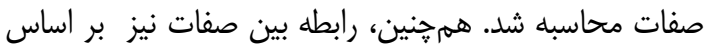

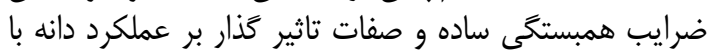
استفاده از تجزيه رَّرسيون كام به كام و و تجريه عليت كاريت تعيين

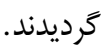

برآورد شد (سا). همجنين مقدار وراثتيذيرى و بازده زنتيكى

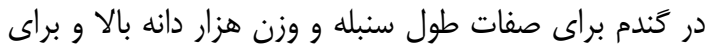

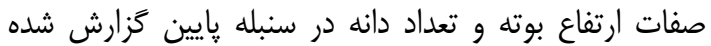

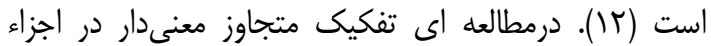

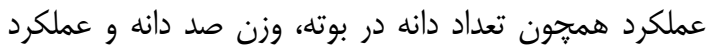

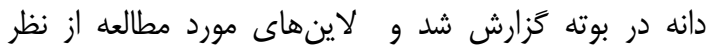

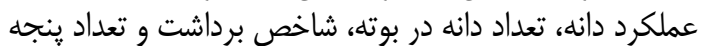

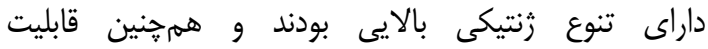

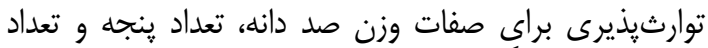

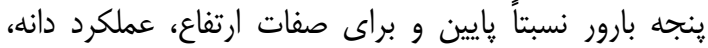

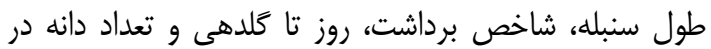

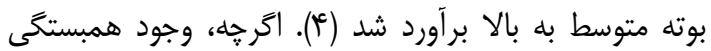

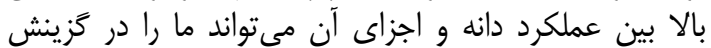

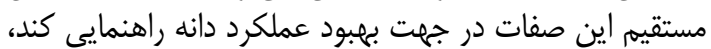

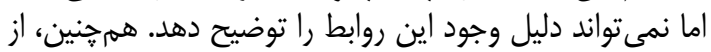

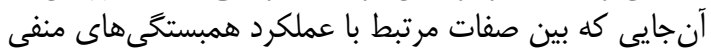

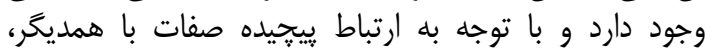

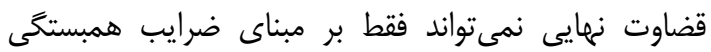

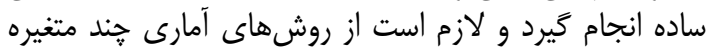

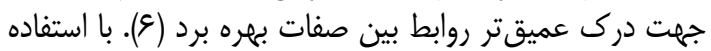

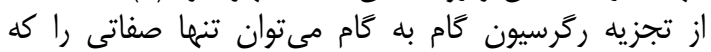

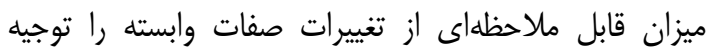

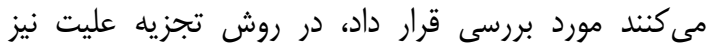

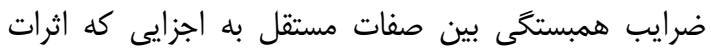
مستقيم و غيرمستقيه را بر روى صفات مفات وابسته اندازهكيرى

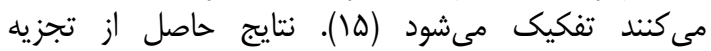

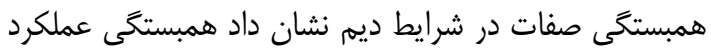

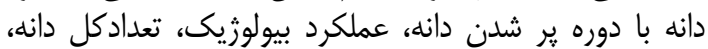

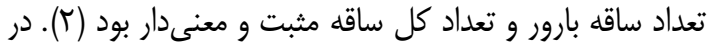

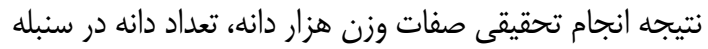

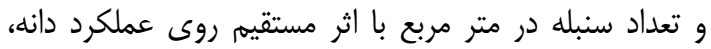

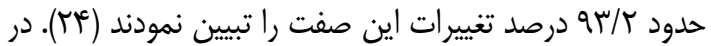

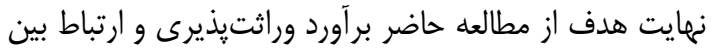

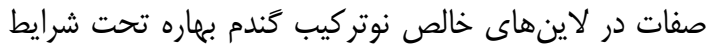

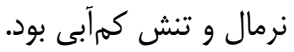

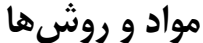

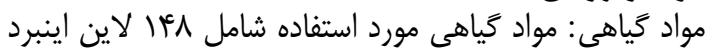

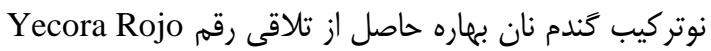

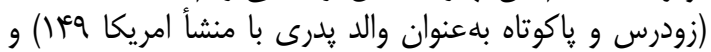

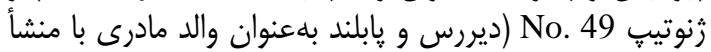

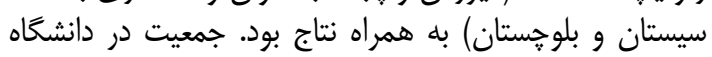

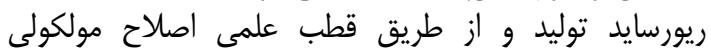

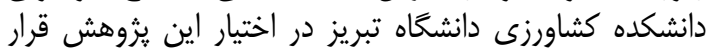

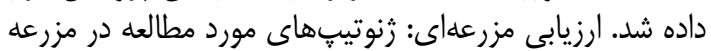

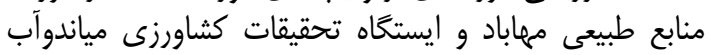

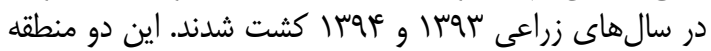

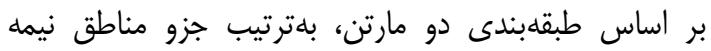
خشى كشور طبقلبندى شدهاند. آزمايش در هر دو منطقي در در 
يدانكل، طول سنبله، تعداد سنبله در متر مربح، وزن سنبله

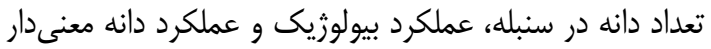

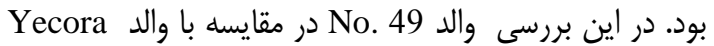

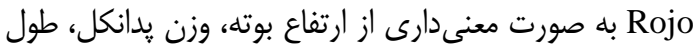

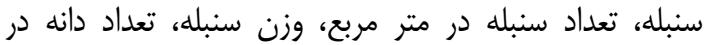

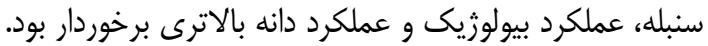

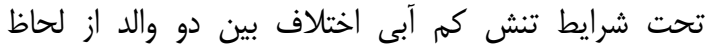

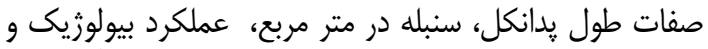

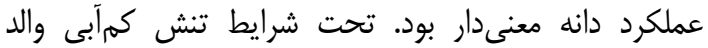

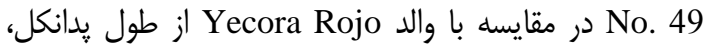

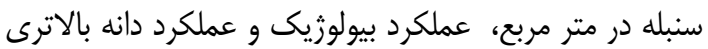

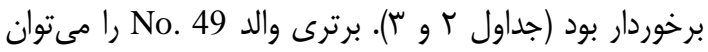

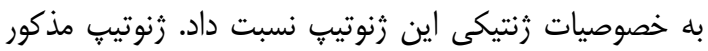

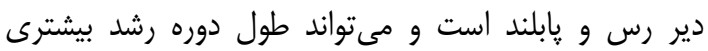

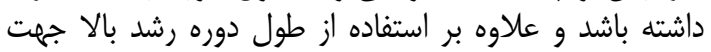

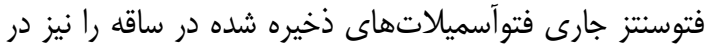

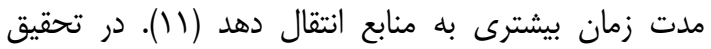

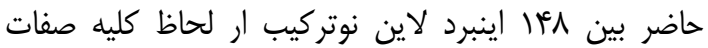

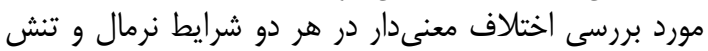

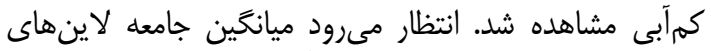

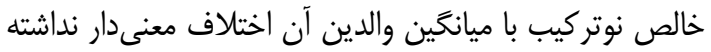

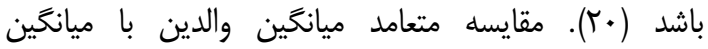

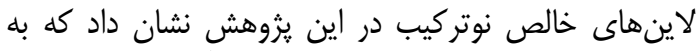

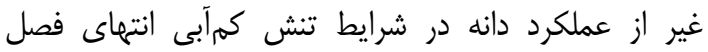

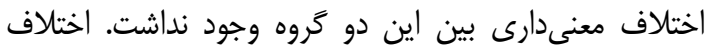

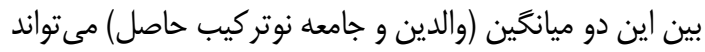

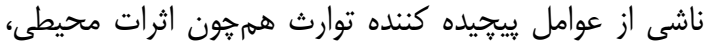

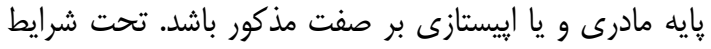

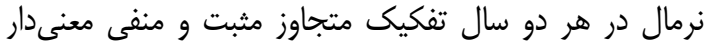

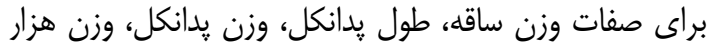

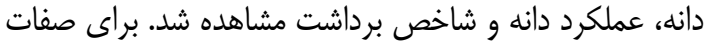

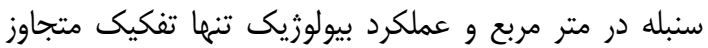

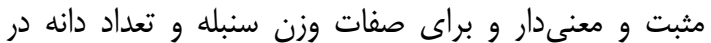

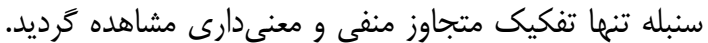

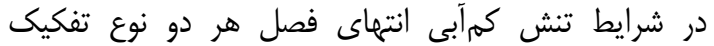

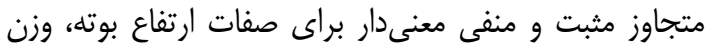

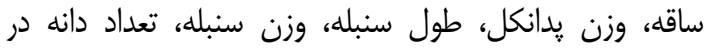

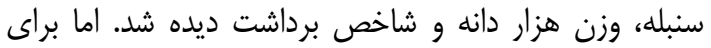

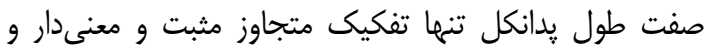

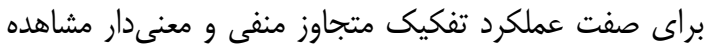

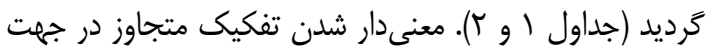

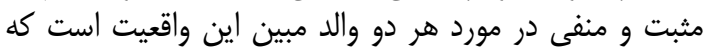

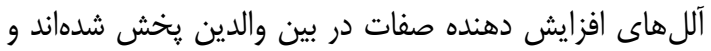

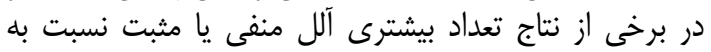

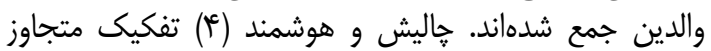

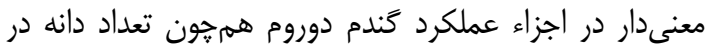

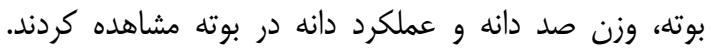

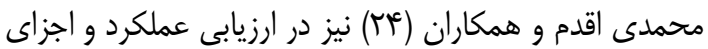
عملكرد در جمعيت لاينهاى اينبرد نوتركيب كندم نان اندان حاصل

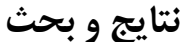 تجزيه واريانس مركب صفات}

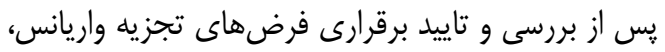

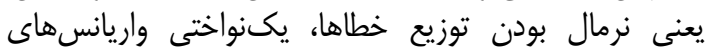

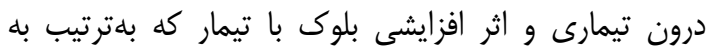

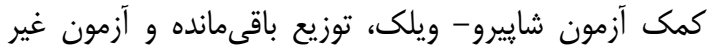

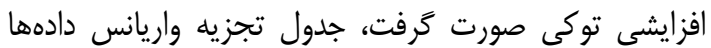

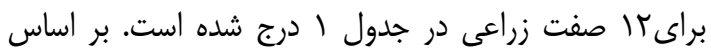

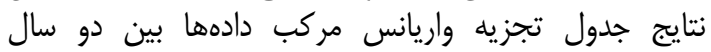

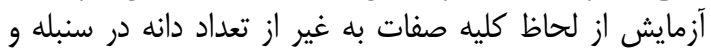

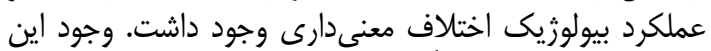

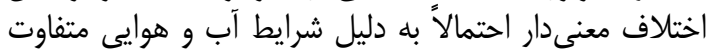

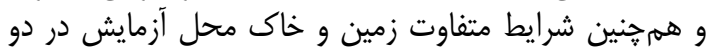

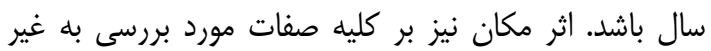

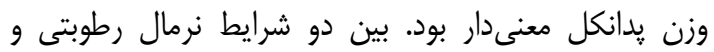

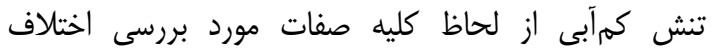

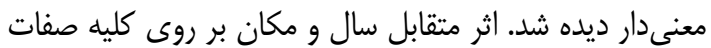

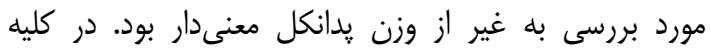

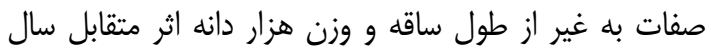

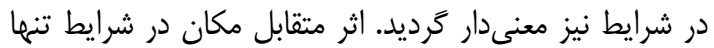

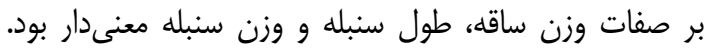

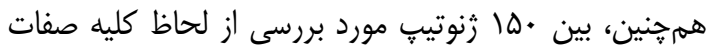

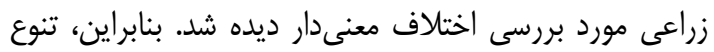

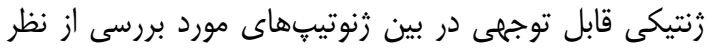

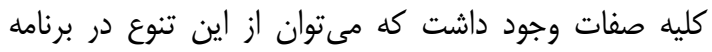

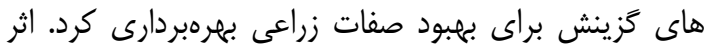

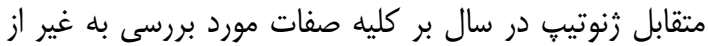

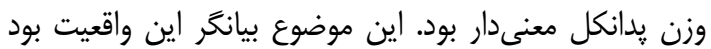

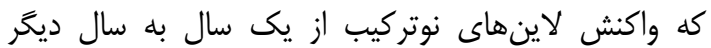

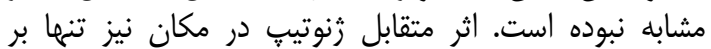

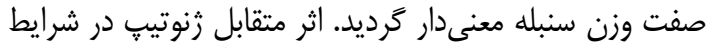

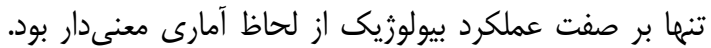

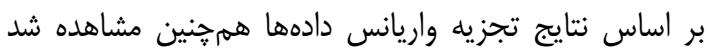

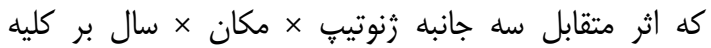

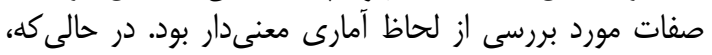

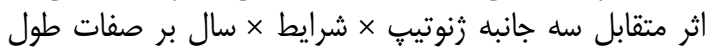

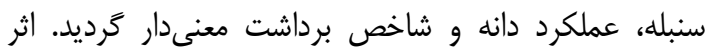

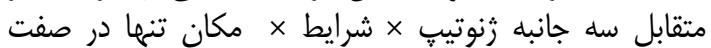

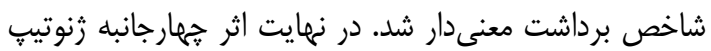

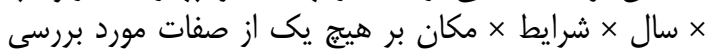

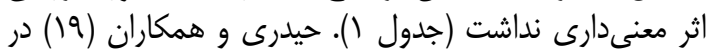

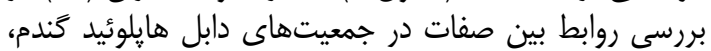

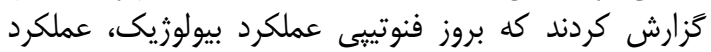

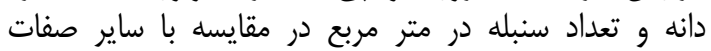

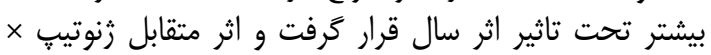

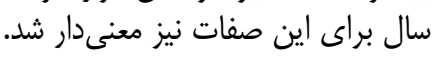

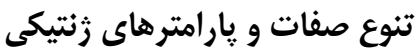
نتايج نشان داد كه در شرايط نرمال اختاف انتاف بين والدهاى Yecora Rojo 
وراثتيذيرى خصوصى محاسبه شده براى صفات طول سنبله،

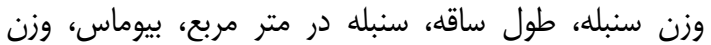

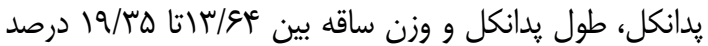

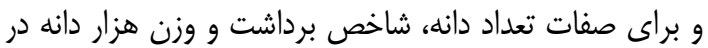

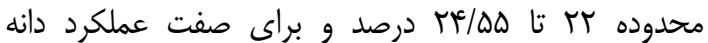

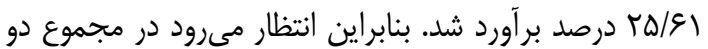

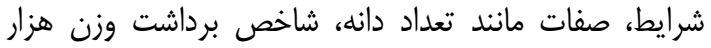

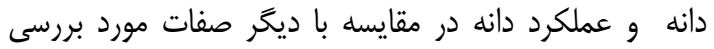

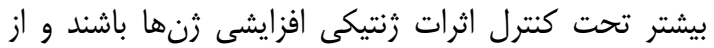

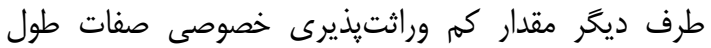

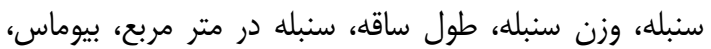

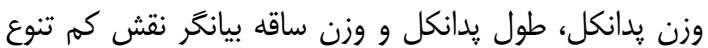

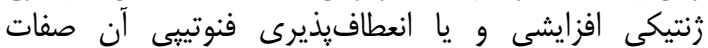

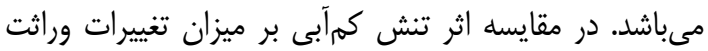

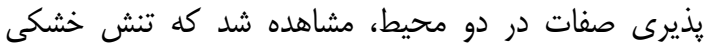
مقدار وراثتيذيرى را در مقايسه با شرايط نرمال در صناه صنات

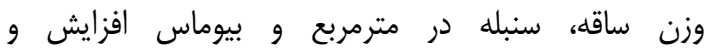

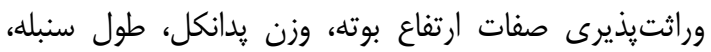

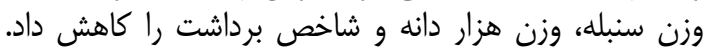

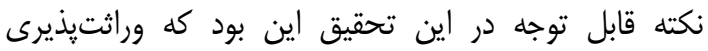

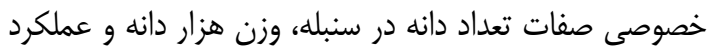

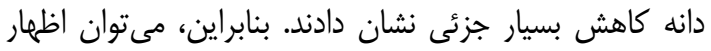

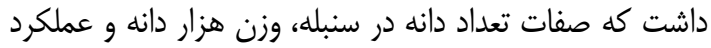

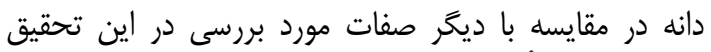

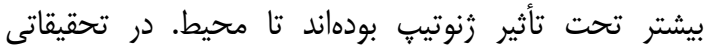

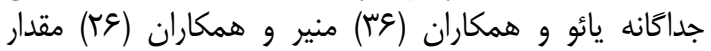

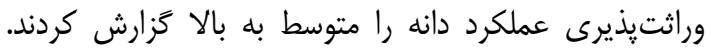

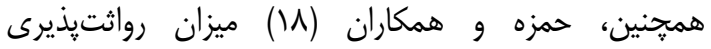

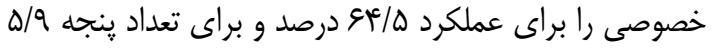

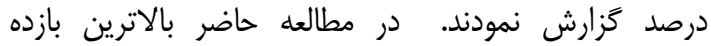

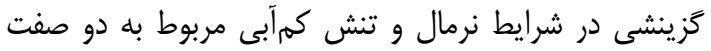

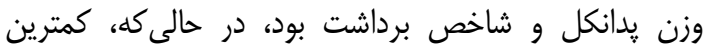

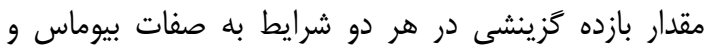

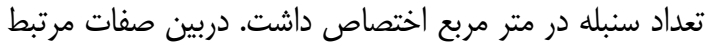

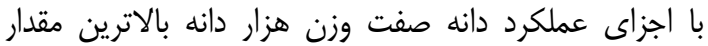

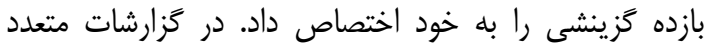

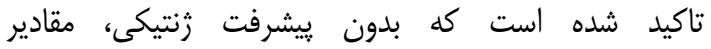

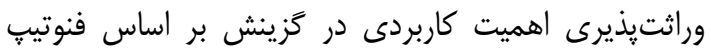

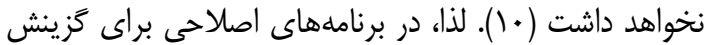

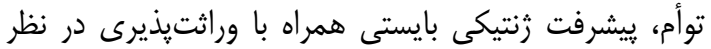

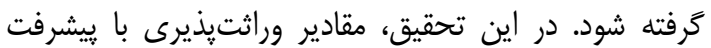

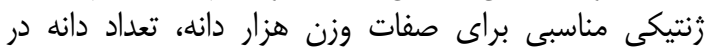

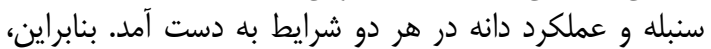

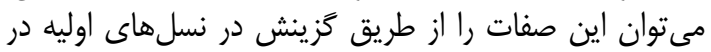

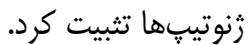
همبستكى بين صفات تُبيت كرد

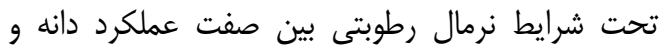

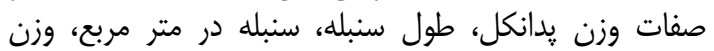
سنبله، تعداد دانه، عملكرد بيولوزيك و شاخلئ در متر برداشت
از تلاقى ارقام زاكرسو نورستار براى صفات عملكرد دانه، تعداد

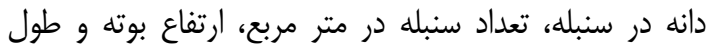

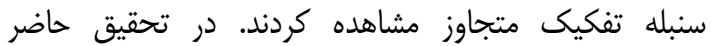

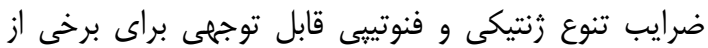

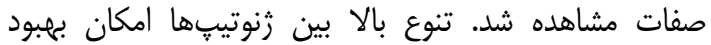

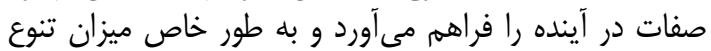

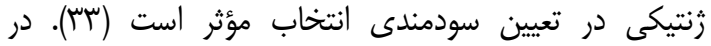

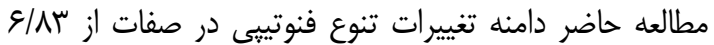

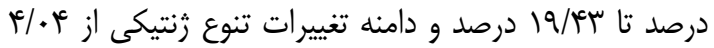

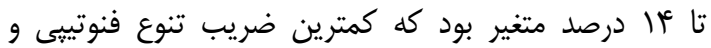

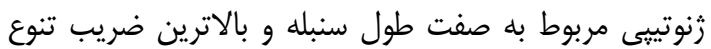

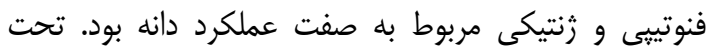

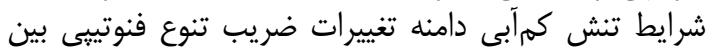

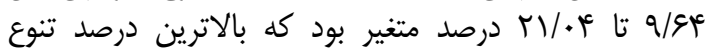

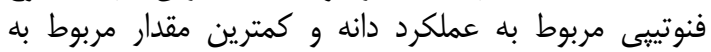

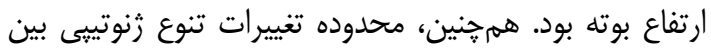

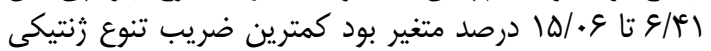

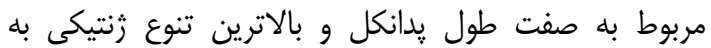

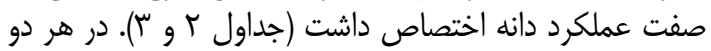

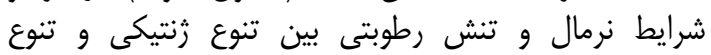

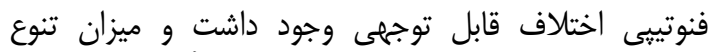

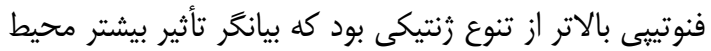

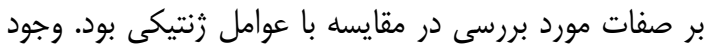

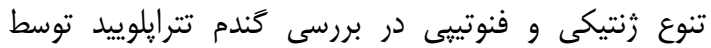

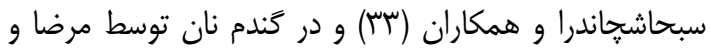

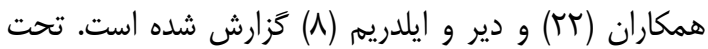

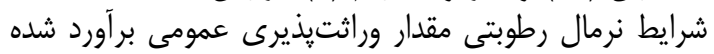

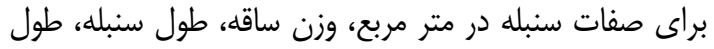

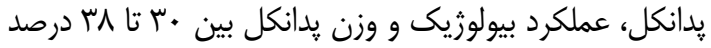

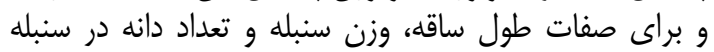

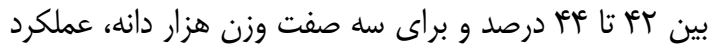

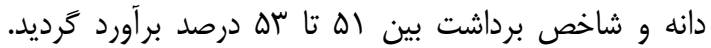

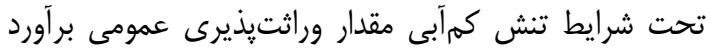

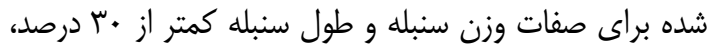

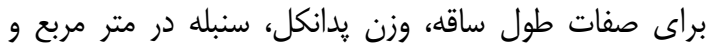

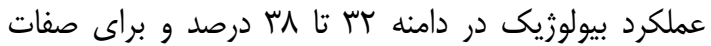

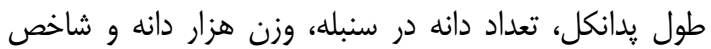

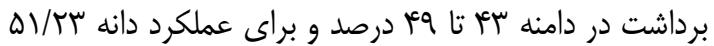

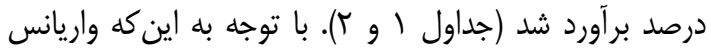

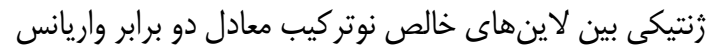

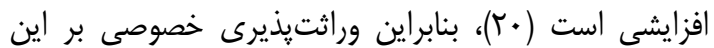

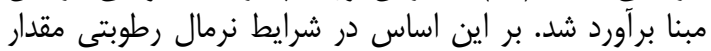

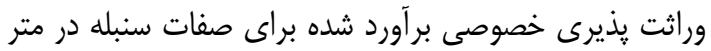

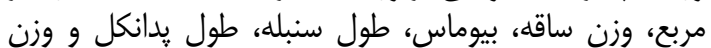

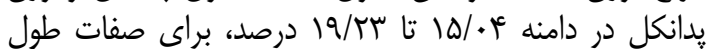

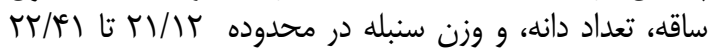

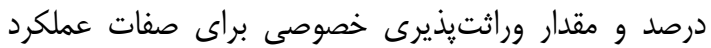

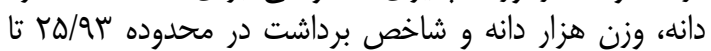

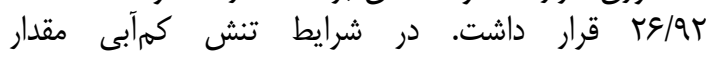


صورت غيرمستقيه و از طريق يكديكر اثر غيرمستقيم منفى بر

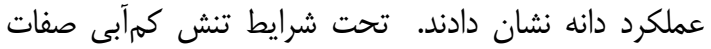

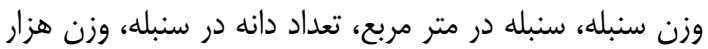

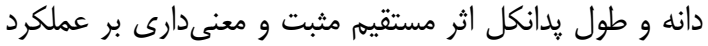

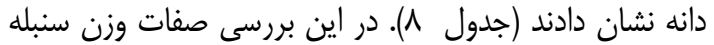

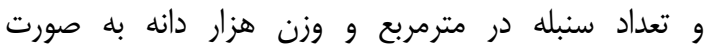

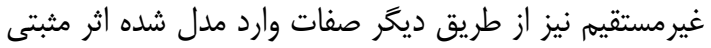

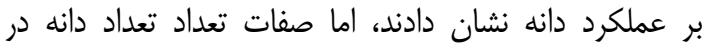

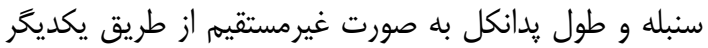

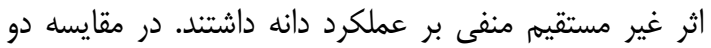

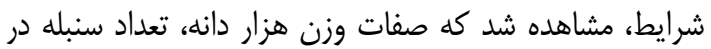

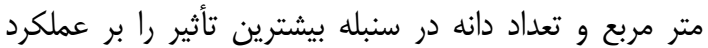

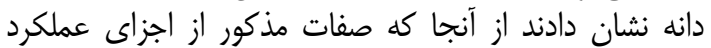

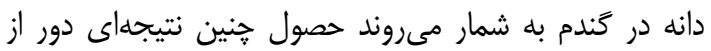

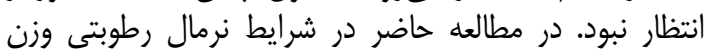

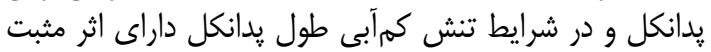

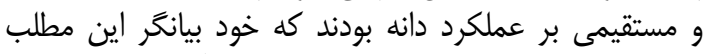

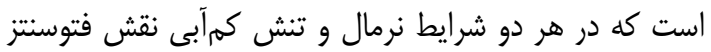

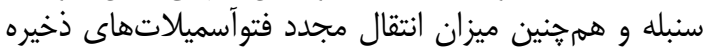

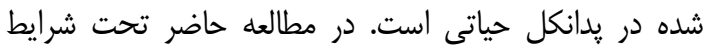

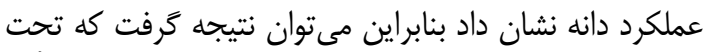

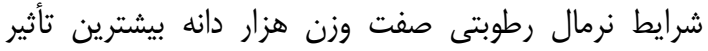

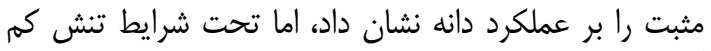

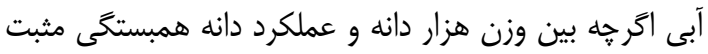

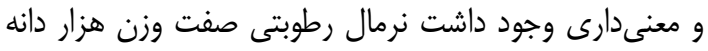

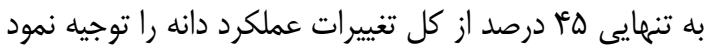

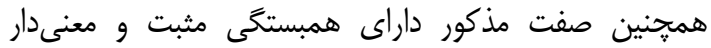

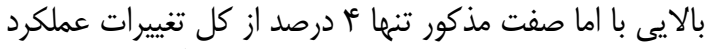

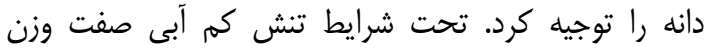

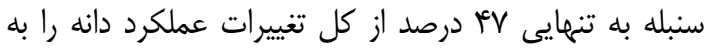

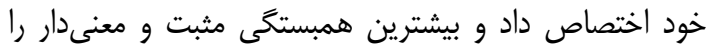

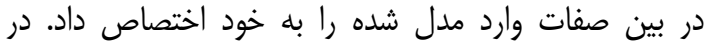

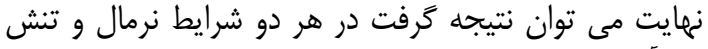

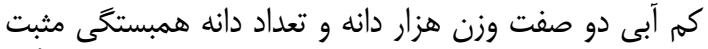

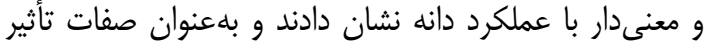

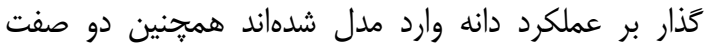

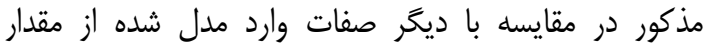

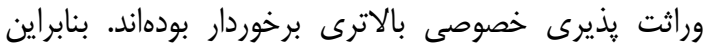

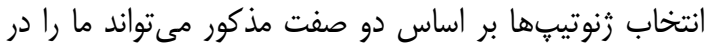

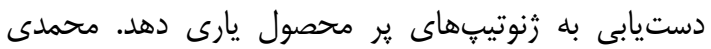

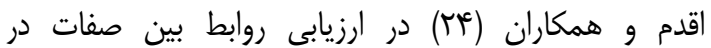

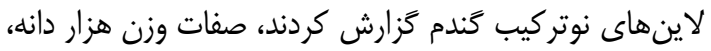

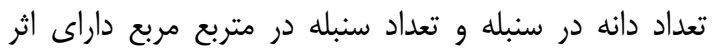

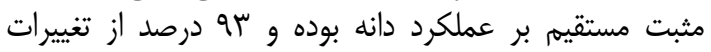
عملكرد دانه را تببين نمودند.

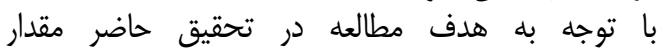

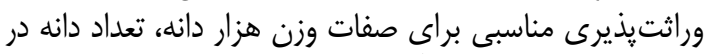

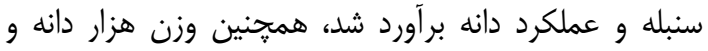
تعداد دانه در سنبله همبستخى مثبت و معنى دار با عملكرد دانه
همبستكى مثبت و معنى دارى ديده شد (جدول كأ أ). تحت

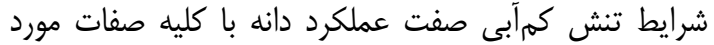

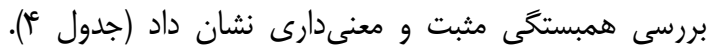

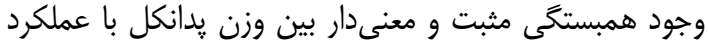

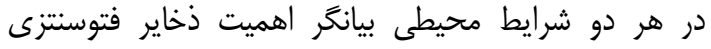

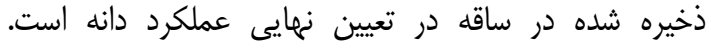

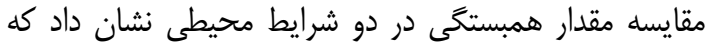

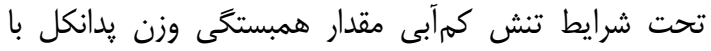

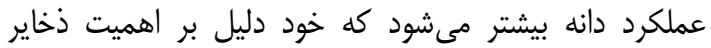

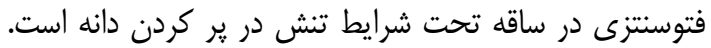

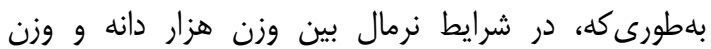

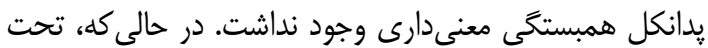

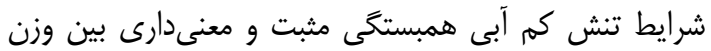

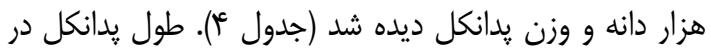

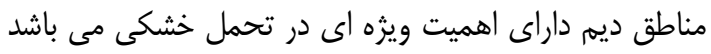

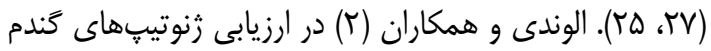

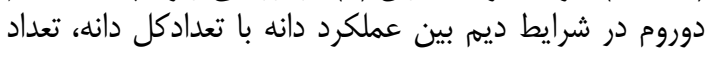

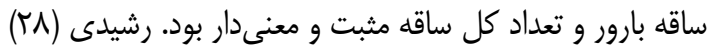

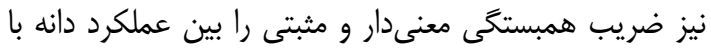

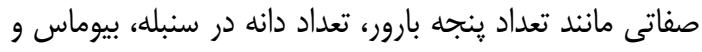
شاخص برداشت كزارش كردان تجزيه رترسيون و عليت

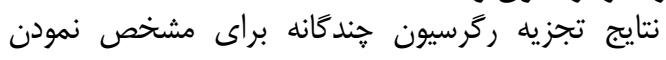

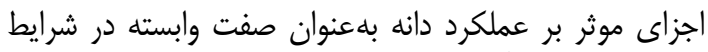

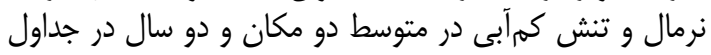

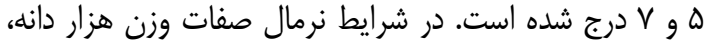
تعداد سنبله در مترمربع، تعداد دانه در در سنبله

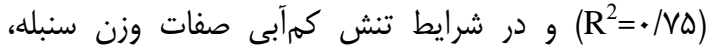

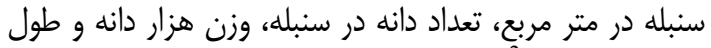

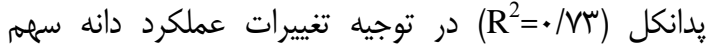

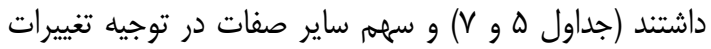

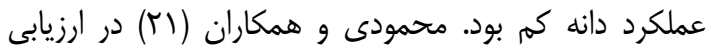

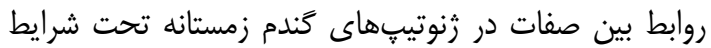

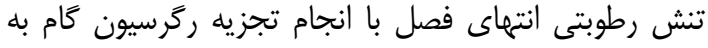

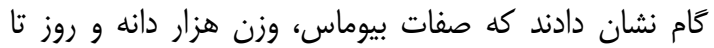

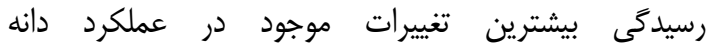
(R2=VV/T\%)

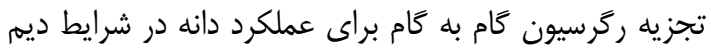

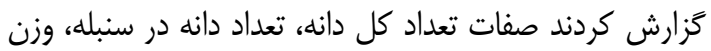

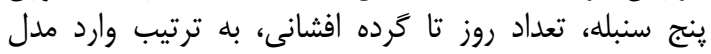

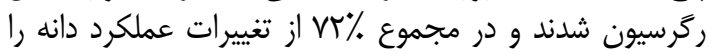

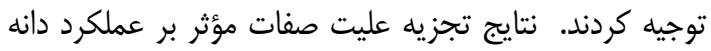

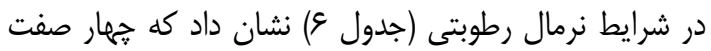

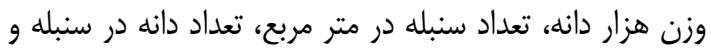

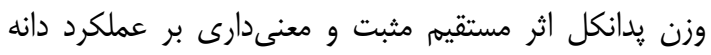

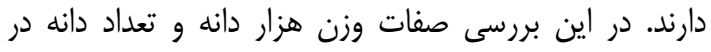

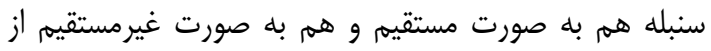
طريق ديخر صفات موجود در مدل اثر مثبتى بر مبر عملكرد دانه نشان دادند. اما دو صفت سنبله در مترمربع و وزن بد يدانكل به ديه 
نرمال و تنش كمابَ آنتهاى فِ فصل بر اساس صفات وزن هزار

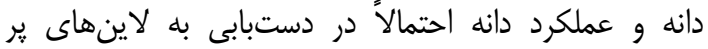
محصول مثمر ثمر باشد.
نشان دادند و بر اساس نتايج تجزيه عليت دو صفت مذكور اثر ماني

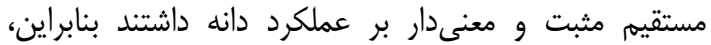
كزينش لاينهاى خالص نوتركيب كندم بهارد تحت دانه شرايط

جدول ا- تجزيه واريانس مركب صفات مورد مطالعه در ^با| اينبرد لاين نوتركيب حاصل از تلاقى دو والد (No. 49 × Yecora Rojo) در دو سال دو مكان و دو شرايط مركب

Table 1. Combined variance analysis of the studied traits in148 recombinant inbred lines derived from the cross of two parents (Yecora Rojo $\times$ No. 49) in two places and two two-year

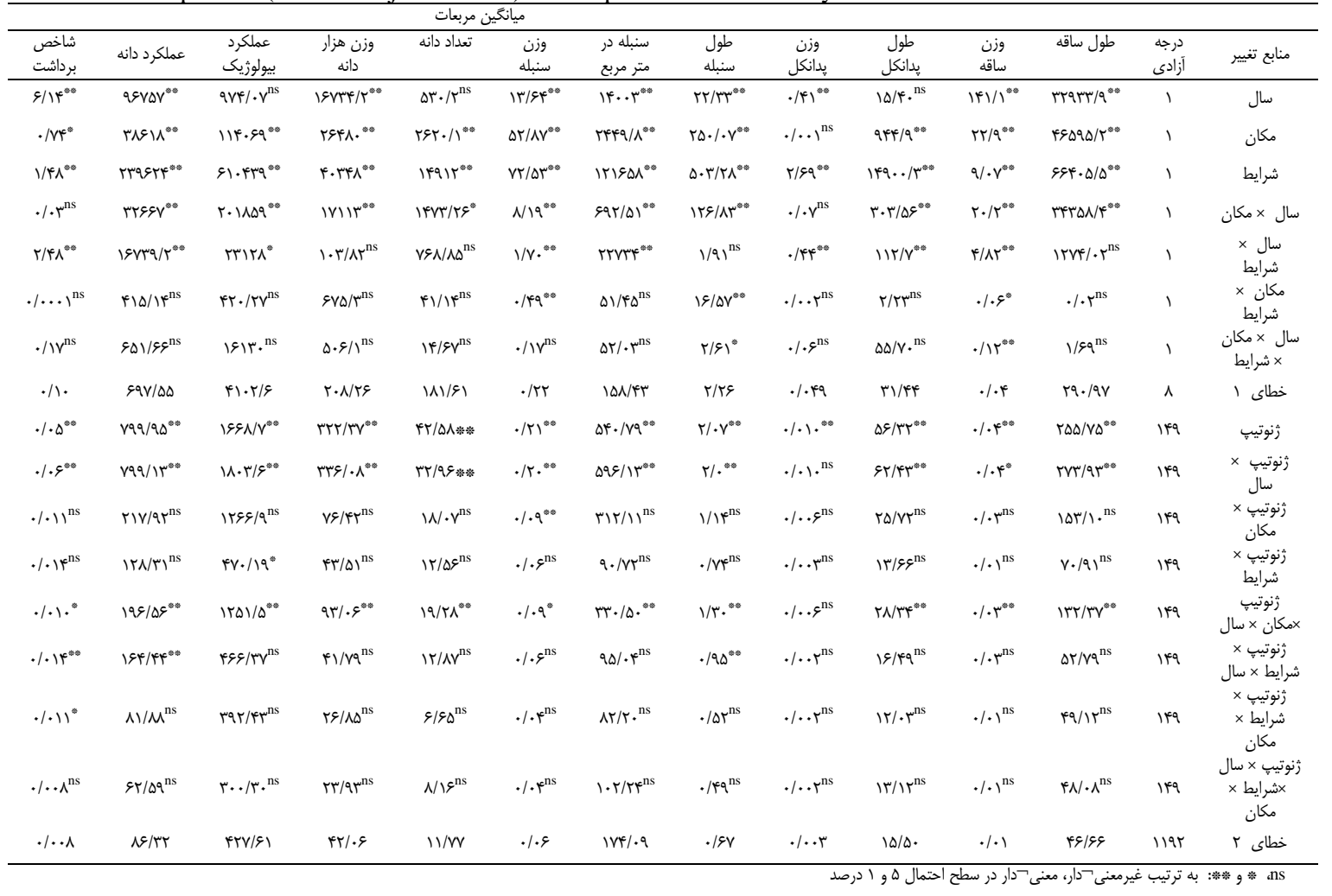

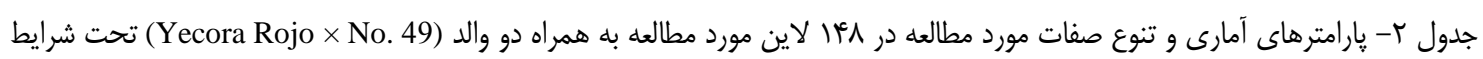

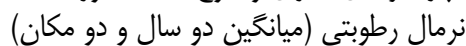

Table 2. Statistical parameters and diversity of understudied traits in 148 studied lines with two parents (Yecora Rojo $\times$ No. 49) under normal irrigation condition (means of two years and two locations)

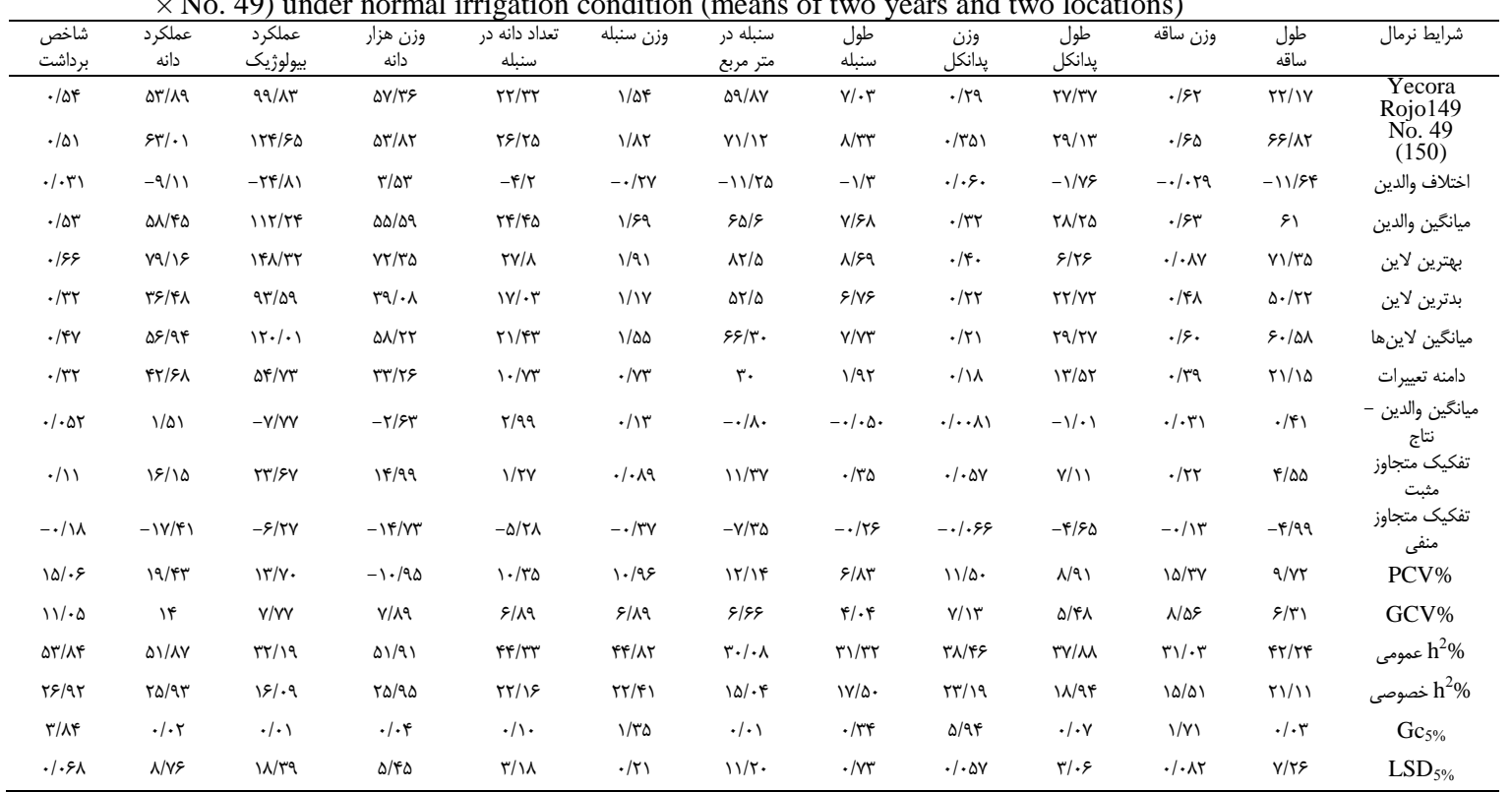


جدول ب- يارامترهاى آمارى و تنوع صفات مورد مطالعه بين ^عا الاين مورد مطالعه به همراه دو والد (Yecora Rojo × No. 49) تحت شرايط

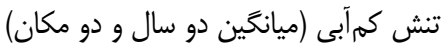

Table 3. Statistical parameters and diversity of understudied traits in 148 studied lines with two parents (Yecora Rojo

\begin{tabular}{|c|c|c|c|c|c|c|c|c|c|c|c|c|}
\hline برداشت & $\begin{array}{l}\text { دمله } \\
\text { د } \\
\end{array}$ & بيولوزيك & وزن هزار & تعداد دانه در & سنبله & سنبله در متر & سول & بديانكل & طبد طول & ساقه & ارتفاع & شرايط نرمال \\
\hline . $/ \Delta T$ & $|N / 9|$ & $V \pi / T V$ & $19 / .1$ & $1 / 199$ & $1 / 19$ & FT/M & 9/99 & r & $r \cdot / A \Delta$ & $\cdot / 44$ & $\mathrm{FV} / \mathrm{QV}$ & $\begin{array}{c}\text { Yecora } \\
\text { Rojo149 }\end{array}$ \\
\hline . $/ \Delta T$ & $F N / N$ & 98 & $\Delta \cdot / A V$ & W/R & $1 / r$. & GI/TA & $\mathrm{V} / \mathrm{M}$ & $\cdot / F^{\prime}$ & 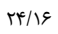 & $.1 .+\Delta$ & D./9T & No. 49 (150) \\
\hline.- .1 .1 & $-1 \cdot / \pi \Delta$ & $-r \cdot \mid g^{\mu}$ & $-1 / N$ & -.1 .4 &.$- / . r$ & $-10 / \mu \Lambda$ &.$- / 0 T$ &.- .1 .1 & ו ו"r/r- &.$- / . \mathrm{r}$ & $-r / 9 \Delta$ & 'اختلاف والدين \\
\hline . $/ \Delta \mu$ & $\kappa r / N$ & $\wedge r / 99$ & $p q / q F$ & $|N / r|$ & $1 / 1 \Lambda$ & $\Delta \Gamma / \Delta S$ & $9 / 9 T$ & 每 & $T r / \Delta)$ &.$/ 4 F$ & $k q / F 4$ & ميانكين والدين \\
\hline . & $\Delta T / \Delta V$ & $11 F / \Delta q$ & $94 / \cdot 1$ & $r .199$ & $1 / 4 \Delta$ & $s \Phi / T \Delta$ & $V / 99$ & & TN/VG &.$/ 8 \Lambda$ & 9.11. & بهترين لاين \\
\hline 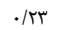 & $\mid V / . t^{\circ}$ & $s \propto / \Delta \Lambda$ & $r / .9$ & $1 r / v g$ &.$/ 91$ & ra/q & $\Delta / \& \Lambda$ & .119 & $W / \mp A$ &.$/ \pi \Delta$ & $r q / \leftarrow \wedge$ & بدترين لاين \\
\hline س & rg/AV & $\Delta V / \Delta V$ & $19 / 9 \Delta$ & $19 / \pi q$ & $1 / M$ & $\Delta \cdot|\lambda|$ & $s / V^{E}$ &.$/ \pi f$ & $\pi / \% \Delta$ &.$/ 4 \Lambda$ & $\Delta \cdot / \Delta$. & ميانكين لاينها \\
\hline.$/ 4$ & r & 0.1 .1 & $r y / \cdot r$ & v/a. &.$/ D F$ & TN/G & $1 / 91$ &.$/ 19$ & $1 . / 79$ &.$/ M_{F}$ & $r . / 9 T$ & دامنه تعييرات \\
\hline.$- / 1$ & $-g / N E$ & $r / M$ & $\%$ & $-1 / \wedge \Delta$ & $\%$ & $-t / V G$ &.$- / 1 \wedge$ & .1 .1 & $1 / 1 f$ & /.r & $1 / .9$ & ميانكين والدين - \\
\hline .1 .9 & $F / V T$ & $r \cdot 1 \Delta A$ & $|r / r|$ & $T / F F$ &.$/ T \Delta$ & r &.$/ 4 \Lambda$ & $.1 \cdot 1$ & $F / 8$. & r & $9 / \backslash \wedge$ & تفكيك متجاوز \\
\hline.$- / \mu$. & $-r / \Delta S$ & 㑩 & $-10 / 9 \Delta$ & $-\Delta / \kappa \mu$ &.$/ T \Delta$ & $-1 \cdot / \pi \Delta$ &.$- / 91$ &.$- / \cdot v$ & 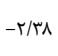 & -.1 .9 & $-1 / F q$ & تفكيك متجاوز \\
\hline IV/NE & $r M / \cdot \varphi^{c}$ & $\mid \mathrm{V} / \cdot \mathrm{V}$ & $1 . / V T$ & $11 / \Delta F$ & $1 . / \Delta T$ & $1 \% / v \Delta$ & $V / 4)$ & IT/N & $1 . / 94$ & $11 / 94$ & $9 / 94$ & PCV\% \\
\hline $11 / 94$ & $10 / .9$ & $9 / \wedge$. & $V / \Delta \mid$ & $V / \& V$ & $\Delta / V \Delta$ & $\mathrm{V} / \mathrm{Ar}$ & $r / A V$ & $N / \cdot r$ & $9 / 41$ & $V E / T F$ & $V / r F^{e}$ & GCV\% \\
\hline 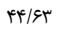 & WI/T & $r r / q \Lambda$ & $k q / 11$ & $\kappa * / .1$ & $r q / \Lambda$. & $r T / 4 F$ & $T V / T \Lambda$ & $r r / 4 V$ & $r r / \Delta r$ & $r / v 1$ & TI/sQ & 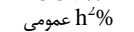 \\
\hline Tr/TI & $r \Delta / \gamma^{\prime}$ & $18 / 97$ & $r F / \Delta \Delta$ & $\pi$ & $16 / 9$. & $19 / \pi T$ & $1 \pi / 94$ & $19 / \mathrm{VF}$ & $I V / r g$ & $19 / \pi \Delta$ & $\Delta / \Delta V$ & خصوصى h خ \\
\hline$r$ & r & .1 .1 & .1 .4 &.$/ 11$ & $1 / \pi$ &.$/ . r$ & $\cdot / r \Lambda$ & $r / 1 Q$ & .1 .9 & $\Gamma / \Delta \Lambda$ & r & $\mathrm{Gc}_{5 \%}$ \\
\hline $.1 \cdot \mathrm{WV}$ & $9 / \pi \pi$ & $10 / \pi \Lambda$ & $\Delta / 1 V$ & T/FT & $\cdot / / M$ & $1 . / 48$ &.$/ 8$ & و צץ./. & $r / \Gamma$. & $.1 \cdot 1 r$ & $\mathrm{~V} / .9$ & $\mathrm{LSD}_{5 \%}$ \\
\hline
\end{tabular}

جدول ع- همبستخى بين صفات در لاينهاى مورد مطالعه تحت شرايط نرمال (اعداد پايين قطر) و تنش كهآبى (اعداد بالاى قطر)

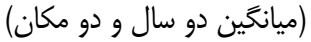

Table 4. Correlation coefficients of traits under normal condition (low numbers), cut irrigation (high numbers) based

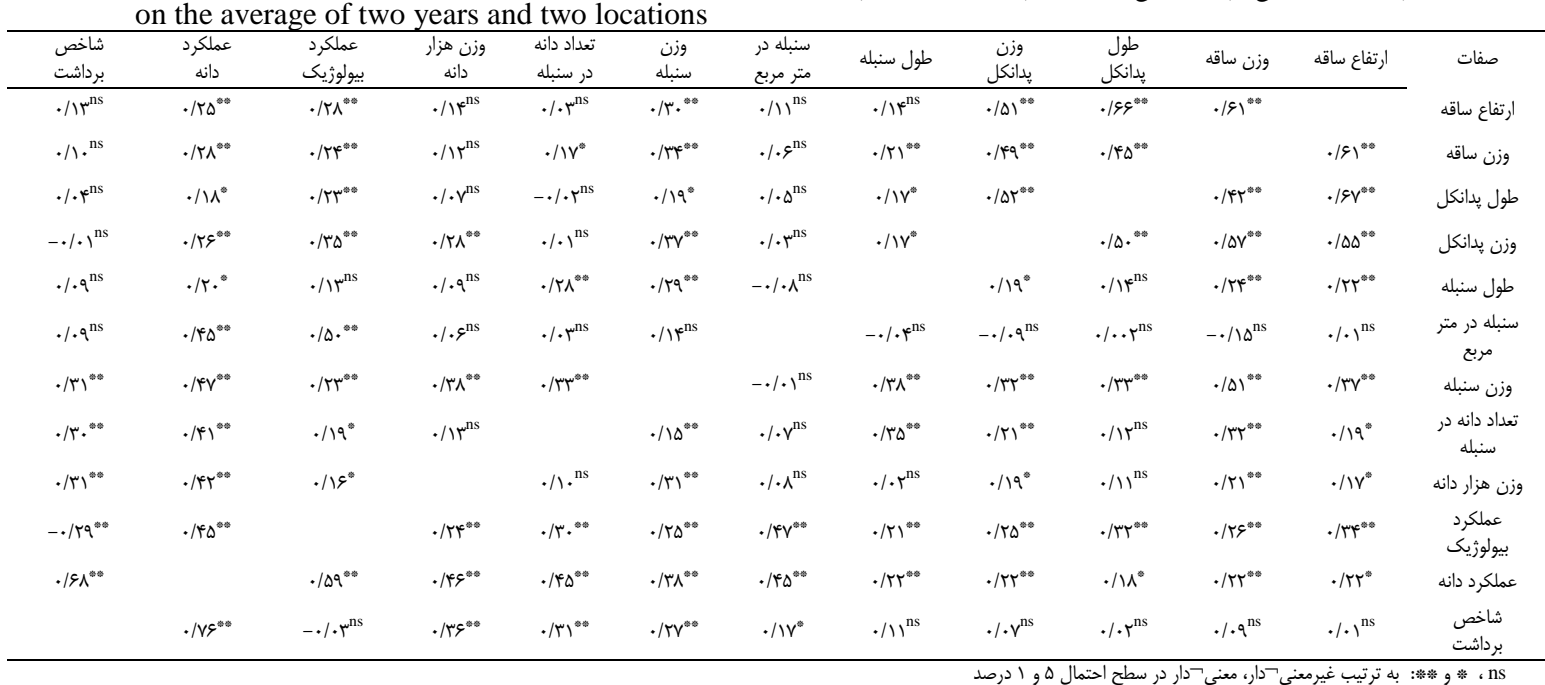

جدول ه- نتايج تجزيه رگرسيون گام به كام صفات مورد مطالعه با عملكرد دانه در شرايط نرمال رطوبتى

Table 5. Results of stepwise regression analysis of under studied traits with grain yield under normal condition

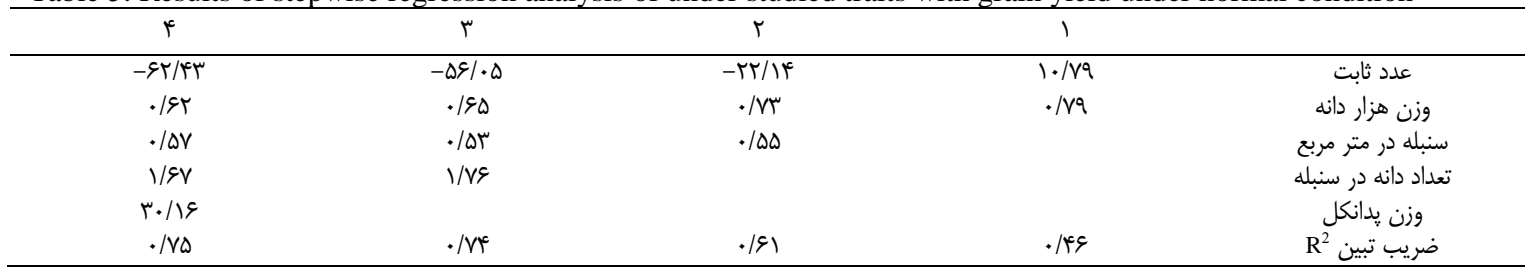


Table 6. path analysis of traits affecting grain yield under normal condition

$$
\text { جدول و- تجزيه عليت صفات مؤثر بر عملكرد دانه در شرايط نرمال رطوبتى }
$$

\begin{tabular}{|c|c|c|c|c|c|}
\hline \multicolumn{6}{|c|}{ اثر غير مستقيم } \\
\hline وزن يدانكل & تعداد دانه در سنبله & سنبله در متر مربع & وزن هزار دانه & اثر مستقيم & \\
\hline$\cdot / \cdot r \cdot$ & $\cdot / \cdot \mu \Lambda$ & 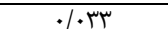 & - & 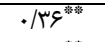 & وزن هزار دانه \\
\hline$-. .1 . .9$ & $.1 \cdot r q$ & - & $.1 \cdot \mathrm{TA}$ & $\cdot / \kappa^{2}$ & سنبله در متر مربع \\
\hline תז./. & - & ./. rq & عץ.|. & 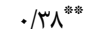 & تعداد دانه در سنبله \\
\hline- & $\cdot 1 \cdot \mathrm{Vq}$ & $-\cdot / \cdot r V$ & .1 .94 &.$/ 11^{*}$ & وزن پِانكل \\
\hline
\end{tabular}

جدول V- نتايج تجزيه رگرسيون گام به كام صفات مورد مطالعه با عملكرد دانه در شرايط تنش كم آبى Table 7. Results of stepwise regression analysis of under studied traits with grain yield under water deficit condition

\begin{tabular}{|c|c|c|c|c|c|}
\hline$\Delta$ & f & $r$ & $r$ & 1 & عدد ثابت \\
\hline - & $-r \Delta / q 1$ & $-M M / V^{2}$ & $-1 T / 1$. & $\Delta / \wedge)$ & وزن سنبله \\
\hline 1./ra & IT & $1 V / 91$ & $r M / \Delta \Lambda$ & DL & سنبله در متر مربع \\
\hline . Rt & $\cdot|k|$ & . kt & $\cdot|+|$ & & تعداد دانه در سنبله \\
\hline I/T & $1 / \cdot 9$ & $1 / .99$ & & & وزن هزار دانه \\
\hline & $\cdot / 4 q$ & & & & طول يدانكل \\
\hline \multicolumn{6}{|l|}{$\cdot / \mu$} \\
\hline$\cdot / r^{m}$ &.$/ V r$ & $.19 \Lambda$ &.$|9|$ &.$/ 4 V$ & ضريب تبين R \\
\hline
\end{tabular}

$$
\text { جدول ^- تجزيه عليت صفات مؤثر بر عملكرد دانه در شرايط تنش كم آبى }
$$

\begin{tabular}{|c|c|c|c|c|c|c|}
\hline طول يدانكل & وزن هزار دانه & تعداد دانه در سنبله & سنبله در متر مستقيم & وزن سنبله & اثر مستقيم & صفات \\
\hline$\cdot 1 \cdot T F$ & $\cdot / 1 \cdot r$ & .1 .99 & $\cdot / \cdot+\wedge$ & - & $\cdot / 1 \Lambda^{2}$ & وزن سنبله \\
\hline $.1 . .9$ & .1 .18 & $.1 . .9$ & - &.$|\cdot 4|$ & $\cdot / 4 \cdot{ }^{*}{ }^{*}$ & سنبله در متر مربع \\
\hline$-\cdot / \cdot \cdot r$ & o & - & $\cdot 1+1$ & $.1 \cdot 09$ & . & تعداد دانه در سنبله \\
\hline $.1 . .9$ & - & ./. एq & $.1 \cdot r f$ & .1 .91 & $\cdot / T V^{* * * 6}$ & وزن هزار دانه \\
\hline- & $.1 \cdot 11$ & $-\cdot 1 \cdot \cdot 9$ &.$/ . r$ & (-l. & ./M" & طول يدانكل \\
\hline
\end{tabular}

Table 8. path analysis of traits affecting grain yield under water deficit condition

1. Abdel-Ghany, H.M. A.A. Nawar M.E Ibrahim, A. El-Shamarka, M.M. Selim and A. Fahmi 2004. Using tissue culture to select for drought tolerance in bread wheat. Proceedings of the $4^{\text {th }}$ International Crop Science Congress Brisbane, Australia, 1- 26.

2. Alvandi, R., A.R .Etminan, R. Mohammadi and L. Shoahtari. 2015. Genetic Diversity of Durum Wheat Genotypes Using Agronomic Characteristics and Molecular Markers. Seed and Plant Improvement Journal, 31: 458-441 (In Persian).

3. Arshad, Y. and A. Soltani. 2006. Effects of drought stress on wheat accessions in gene banks. the final report. Registration number 85.1239 Agricultural Information and Documentation Centre, 80 pp (In Persian).

4. Chalish, L. and S. Houshmand. 2013. Estimate of Heritability and Relationship of Some Durum Wheat Characters Using Recombinant Inbred Lines. Electronic Journal of Crop Production, 4(2): 223238 (In Persian).

5. Chandra, D., M.A. Islam and N.C.D. Barma. 2004. Variability and interrelationship of nine quantitative characters in $\mathrm{F}_{5}$ bulks of five wheat crosses. Pakistan journal Biology Since, 6: 10401045.

6. Cooper, J.C.B. 1983. Factor analysis. An overview. The Amerian Statistician, 37(2): 141-147.

7. Dawari, N.H. and O.P. Luthra. 1991. Character association studies under high and low environments in wheat. Indian Journal of Agricultural Research, 25: 515-518.

8. Dere, S. and M.B. Yildirim. 2006. Inheritance of grain yield per plant, flag leaf width and length in an 8 x 8 Diallel cross population of bread wheat (T. aestivum L.). Turk journal of agricultural, 30: 339345 .

9. Ehdaei, B. and A. Ghaderi. 1972. Diallel method and its application in plant breeding. Shahid Chamran University Press, 54 pp (In Persian).

10. Ehdaie, B. and J.G. Wains. 1997. Genetic analysis of carbon isotope discrimination and agronomic characters in a bread wheat cross. Theoritical and Applied Genetics, 88(8): 1023-1028.

11. Ehdaie, B., G.A. Alloush and J.G. Waines. 2008. Genotypic variation in linear rate of grain growth and contribution of stem reserves to grain yield in wheat. Field Crops Research, 106: 34-43.

12. Eid, M.H. 2009. Estimation of heritability and genetic advance of yield traits in wheat (Triticum aestivum L.) under drought condition. International Journal of Genetics and Molecular Biology, 1(7): 115-120.

13. Erkul, A., A. Ünay and C. Konak. 2010. Inheritance of yield and yield components in a bread wheat (Triticum aestivum L.) cross. Turkish Journal of Field Crops, 15(2): 137-140. 
14. FAO. 2012. FAOSAT agricultur data. Agricultural production 2009. FAO. Rome. Fao. Org. Accessed 22 Apr 2012.

15. Farshadafar E. 2005. Principles and multivariate statisticalmethods (second edition). Kermanshah, Publications Taq Bostan, 734 pp (In Persian).

16. Fethi, B. and E.G. Mohamed. 2010. Epistasis and genotype-by-environment interaction of grain yield related traits in durum wheat. Plant Breeding and Crop Science, 2(2): 24-29.

17. Gol-Abadi, M., A. Arzani and S.A.M. Mirmohammady Maibody. 2008. Genetic analysis of some morphological traits in durum wheat by generation mean analysis under normal and drought stress conditions. Seed Plant, 24: 99-116 (In Persian).

18. Hamze. H., J. Saba, F. Jabari, J. Nassiri and M. Alavi. 2008. Estimation of components variation, genotypic and phenotypic correlation coefficients of grain yield and its component in bread wheat (Tritium aestivum L.) under rainfed conditions Environment Stresses in Agriculture Sciences, 2(1): 29-38 (In Persian).

19. Heydari, B., A. Sayed-Tabatabaei Badreddin and A. Said. 2010. The interaction of genotype on the heritability of traits and genetic improvement of wheat doubled haploid choice in a community. Iranian, journal of Agriculture science, 39(1): 41-50 (In Persian).

20. Houshmand, S. 2003. The genetical analysis of quantitative traits. ShahreKord Univ. Pub, $462 \mathrm{pp}$.

21. Mahmoudi, A., S. Mohammadi, J. Sabah, H. Hamza and M. Rezaei. 2014. Evaluation of relationships between traits in winter wheat genotypes under stress, the end of the season. Cereal Research, 4(1): 11-1 (In Persian).

22. Marza, F., G.H. Bai, B.F. Carver and W.C. Zhou. 2006. Quantitative trait loci for yield and related traits in the wheat population Ning $7840 \times$ Clark. Theoritical and Applied Genetics, 112: 688-698.

23. Memon, S.M., B.A. Ansari and M.Z. Balouch. 2005. Estimation of genetic variation for agroeconomic traits in spring wheat (Triticum aestivum L.). Indian Journal of Plant Sciences, 4: 171-175.

24. Mohammadi Aghdam, M.D., A. Nasrollahzadeh and S.A. Mohammadi. 2012. Evaluation yield and yield components of bread wheat recombinant inbred line population derived from the cross, Zagros and Norstar figures. Journal of Agricultural Science and Sustainable Production, 21(4): 39-40 (In Persian).

25. Mohammadi, A., E. Majidi, M.R. Bihamta and H. Heidari Sharifabad. 2006. Evaluation of drought stress on agro-morphological characteristics in some wheat cultivars. Agronomi Journal (Pajouhesh and Sazandegi), 73: 184-192 (In Persian).

26. Munir, M., M. Aslam, M. Chowdhry and M.D. Ashan. 2007. Generation means studies in bread wheat under drought condition. Interernatinal Journal of Agricultural and Biology, 9: 282-286.

27. Murphy, K.M. and P.G. Reeves. 2008. Relationship between yield and mineral nutrient concentrations in historical and modern spring wheat cultivars. Euphytica, Springer Netherlands, 3: 381-390.

28. Rashidi, V. 2011. Genetic parameters of some morphological and physiological traits in durum wheat genotypes (Triticum durum L.). African Journal of Agricultural Research, 6(10): 2285-2288.

29. Rebetzke, G.J., R.A. Richards, A.G. Condon and G.D. Farquhar. 2006. Inheritance of carbon isotope discrimination in bread wheat (Triticum aestivum L.). Euphytica, 150: 97-106.

30. Sharma, J.R. 2006. Statistical and biometrical techniques in plant breeding. New Age. International. Ltd, $429 \mathrm{pp}$.

31. Sharma, S.N., R.S. Sain and R.K. Sharma. 2002. The genetic system controlling number of spikelets per ear in macaroni wheat over environments. Wheat Information Service, 95: 36- 40.

32. Singh, K.N. and R. Chatrath. 1992. Genetic variability in grain yield and its component characters and their associations under salt stress conditions in tissue culture lines of bread wheat (Triticum aestivum L. em Thell.). Wheat information Service, 75: 46-53.

33. Subhashchandra, B., H.C. Lohithaswa, A.S. Desai and R.R. Hanchinal. 2009. Assessment of genetic variability and relationship between genetic diversity and transgressive segregation in tetraploid wheat. Karnataka Journal of Agricultural, 22: 36-38.

34. Taleei, A. and G.H. Nourmohammadi. 1994. Calculation of general and specific inheritability in 3 hybridizations of bread wheat. Iranian Journal of Agricultural Scinces, 25(4): 79-86 (In Persian).

35. Therrien, M.C. 2003. Heritability estimates for forage quality in barley. Barley Genetics Newsletter, 33: $16-17$

36. Yao, J., G.Yao, X. Yang, C. Qian and S. Wang. 2004. Analysis on the combining ability and heritability of the spike characters in wheat. Acta Agriculturae Shanghai, 20: 32-36.

37. Young, N.D. 2000. Construction of plant genetic linkage map with DNA markers, In: R.L. Phyllips and J.K. Vasil, (eds), DNA-Based Markers in Plants. Kluwer Academic Publications, 31- 47 pp.

38. Zarkti, H., H. Ouabbou, A. Hilali and S.M. Udupa. 2010. Detection of genetic diversity in Moroccan durum wheat accessions using agro-morphological traits and microsatellite markers, African Journal of Agricultural Research, 5(14): 1837-1844. 


\title{
Estimates of Heritability and Association among Traits in Recombinant Inbred Lines of Spring wheat under Normal and Terminal Water Conditions
}

\author{
Hamza Hamza ${ }^{1}$, Ali Asghari ${ }^{2}$, Seyed Abulghasem Mohammadi ${ }^{3}$, Omid Sofalian ${ }^{4}$ and \\ Soleyiman Mohammadi
}

1- Ph.D. Student of Biometrical Genetics at the University of Mohaghegh Ardabil Address: PNU center of Mahabad, 2- Associate Professor, University of Mohaghegh Ardabil (Corresponding author: ali_asgharii@uma.ac.ir)

3- Professor, Department of Plant Breeding and Biotechnology, Faculty of Agriculture, Univeristy of Tabriz, Iran 4- Associate Professor, University of Mohaghegh Ardabil

5- Department of Seed and Plant Improvement Research, West Azerbaijan Agricultural and Natural Resources

Research Center, AREEO, Urmia, Iran
Received: August 1, $2016 \quad$ Accepted: January 28, 2018

\begin{abstract}
To estimate heritability and association between traits traits, 148 recombinant inbred lines and their parents (Yecora Rojo and No. 49) were evaluated in Alfa lattice design with two replications under normal and water deficit conditions during two cropping season (2013-15). Results showed that there were significant differences between genotypes for all studied traits. The estimated heritabilities for spikes per square meter, stem weight, biomass, spike length, peduncle length, peduncle weight, shoot length, number of grains per spike and spike weight were in the range of 15.04 to 22.41 and for grain yield, 1000- kernel weight and harvest index were in the range of 25.93 to 26.92 , respectively. Under water deficit conditions, estimated heritabilities for spike length, spike weight, shoot length, spike per square meter, biomass, peduncle weight, peduncle length and stem weight were between 13.64 to 19.35 percent and for number of grains per spike, harvest index, thousand kernel weight and grain yield were in the range of 22 to 25.61 percent. The highest genetic gain in both conditions belongs to peduncle weight and harvest index, respectively. Finally based on regression and path analysis traits of number of grains per spike and 1000- kernel weights were identified as the most effective traits on grain yield in both conditions. Therefore, use of harvest index, grain yield, number of grains per spike and grain weight as indicators of selection, probably can provides access to highyielding lines.
\end{abstract}

Keywords: Correlation, Heritability, Recombinant inbred lines, Wheat bread 